\title{
The Investigation of the Apoptose Structural Effects and Mechanism in Leukemic Cells of Sirt1 Inhibitor Sirtinol
}

\author{
Dilara Fatma Akın1*, Mine Mumcuoğlu², Didem Torun Özkan³, Nejat Akar, \\ Ahmet Emin Kürekçi² \\ ${ }^{1}$ Faculty of Medicine, Medical Biology, Nigde Omer Halisdemir University, Nigde, Turkey \\ ${ }^{2}$ LOSANTE Children's and Adult Hospital, Ankara, Turkey \\ ${ }^{3}$ Vocational School of Health Service, Okan University, İstanbul, Turkey \\ ${ }^{4}$ TOBB-ETU Hospital, Ankara, Turkey \\ Email: *dilarafatmaakin@gmail.com
}

How to cite this paper: Akın, D.F., Mumcuoğlu, M., Özkan, D.T., Akar, N. and Kürekçi, A.E. (2018) The Investigation of the Apoptose Structural Effects and Mechanism in Leukemic Cells of Sirt1 Inhibitor Sirtinol. Open Access Library Journal, 5: e4268. https://doi.org/10.4236/oalib.1104268

Received: December 18, 2017

Accepted: January 26, 2018

Published: January 29, 2018

Copyright $\odot 2018$ by authors and Open Access Library Inc.

This work is licensed under the Creative Commons Attribution International License (CC BY 4.0).

http://creativecommons.org/licenses/by/4.0/

\begin{abstract}
Human SIRT1 is an enzyme that deacetylates the p53 tumor suppressor protein. It has been suggested to modulate p53-dependent functions including DNA damage-induced cell death. Sirtuins are nicotinamide adenine dinucleotide dependent class III histone deacetylase proteins that play a crucial role in several cellular processes, including DNA repair, apoptosis, and lifespan. In this study, we investigated the relationship between sirtinols and apoptosis mechanism at leukemic cells. For this, we applied sirtinol to K-562 (chronic myeloid leukemia) and Jurkat (acute T-cell leukemia) cell lines at different dilutions in cell culture conditions. And Cdna isolated patient RNA samples, after that Caspase3, Bax, Bcl2 expression performed with used qRT-PCR technique and cells were stained by Annexin $\mathrm{V}$ method. According to the results of research, we identified that Sirtinol dilutions were increased in both K-562 and Jurkat, while the number of living cells was decreasing, the number of dead cells increased at $50 \mu \mathrm{M}$ dilution. Sirtinol, an HDAC inhibitor in the direction of these results, has been observed to be a drug that can be effective in the treatment of CML as well as T-ALL.
\end{abstract}

\section{Subject Areas}

Hematology

\section{Keywords}

SIRT1, Sirtinol, Leukemia Cell 


\section{Introduction}

Sirtuin (NAD+) is dependent protein A group of proteins belonging to the family of lysine modifying enzymes. Sirtuins are also known as Class III histone deacetylases (HDACs) and are (NAD+) - dependent, distinguishing them from other group HDACs.

There are 7 different sirtuins (SIRT1-7) in the mammals. Sirtuins play a key role in many different cellular and physiological processes [1]. SIRT1 is mainly a nuclear deacetylase [2]. SIRT1 cleaves acetyl groups in histone and non-histone proteins from lysine residues and controls expression of target genes and protein activities. SIRT1 also plays a role in cell senescence, cell differentiation, apoptosis and cell growth, vasodilatation and angiogenesis stimulating effects [3]. For this reason, excessive expression of SIRT1 may lead to tumor growth and drug resistance. Among the target, genes of SIRT1 are genes that control processes such as proliferation, differentiation, DNA damage and stress response, genomic stability [4] [5].

Sirtinol is a specific SIRT1 inhibitor. Sirtrin has been shown to induce apoptosis in adult T-cell leukemia cells [6]. However, no study investigating the apoptotic effect and mechanism of Sirtinol in Chronic Myeloid Leukemia (CML) cells has been found.

Although many effective SIRT1 inhibitors have been developed so far, very little is known about anti-cancer effects and their mechanisms of action. The aim of this study is to investigate the mechanism of apoptosis in leukemia cells of Sirtinol, which may be a potential anti-cancer drug, primarily CML cells.

\section{Material and Methods}

In the study, K-562-CCL-243 (chronic myeloid leukemia) and Jurkat-TIB-152 (acute T-cell leukemia) cell lines. Cell lines were obtained from ATCC. Leukemic cell lines, K-562 and Jurkat, were grown in RPMI medium (Lonza, Belgium) supplemented with $20 \%$ fetal calf serum (FCS) (Lonza, Belgium), $50 \mathrm{mg} / \mathrm{ml} \mathrm{pe-}$ nicillin/streptomycin and 1\% L-glutamine (Lonza, Belgium). Each cell line was maintained in a humidified incubator at $37^{\circ} \mathrm{C}$ supplied with $5 \% \mathrm{CO}_{2}$. Sirtinol (Sigma-S7942) was applied to K-562 and Jurkat cell lines at different dilutions in cell culture conditions $10 \mu \mathrm{M}, 25 \mu \mathrm{M}$ and $50 \mu \mathrm{M}$. And, only DMSO (Sirtinol's carrier) cells were used as controls.

$4 \times 10^{6}$ cells from each cell line were counted and seeded into $25 \mathrm{~cm}^{2}$ flasks. Then Sirtinol was applied into the flasks at the three different concentrations, 10 $\mu \mathrm{M}, 25 \mu \mathrm{M}$ and $50 \mu \mathrm{M}$. After the incubation with Sirtinol in the time periods of 24 hours experiments were stopped by removing Sirtinol containing medium. Cells were washed once with cold 1xPBS and then pellet were collected by centrifugation to isolate RNA.

RNA isolation was performed with the MagNa Pure Automatic Isolation system (Roche). Caspase3, Bax, Bcl2 expression assays were performed with the qRT-PCR technique and Basic Relative Quantification software using the Light 
Cycler 480 SYBR Green I Master kit and the appropriate protocols from Roche, following the manufacturer's instruction. cDNA was isolated from RNA samples.

These cells were subjected to Annexin-V staining (Annexin-V Apoptosis detection kit, Santa Cruz) after the cells were exposed to the Sirtinol application at the dilutions indicated above and the cells were cultured in Flow cytometry (BD FACS Canto), in order to determine if Sirtinol caused apoptosis.

\section{Results}

Caspase3, Bax and Bcl2 expression assays were performed using real-time PCR from important genes that play a role in apoptosis after application of Sirtinol in different dilutions of K-562 and Jurkat cells (Figure 1).

Caspase 3 expression in K-562 cells decreased at 10 and $25 \mu \mathrm{M}$, but increased above the control level of $50 \mu \mathrm{M}$. In the case of Bcl2 and Bax, there is a decrease in all dilutions compared to the control. Caspase 3 expression in Jurkat cells was
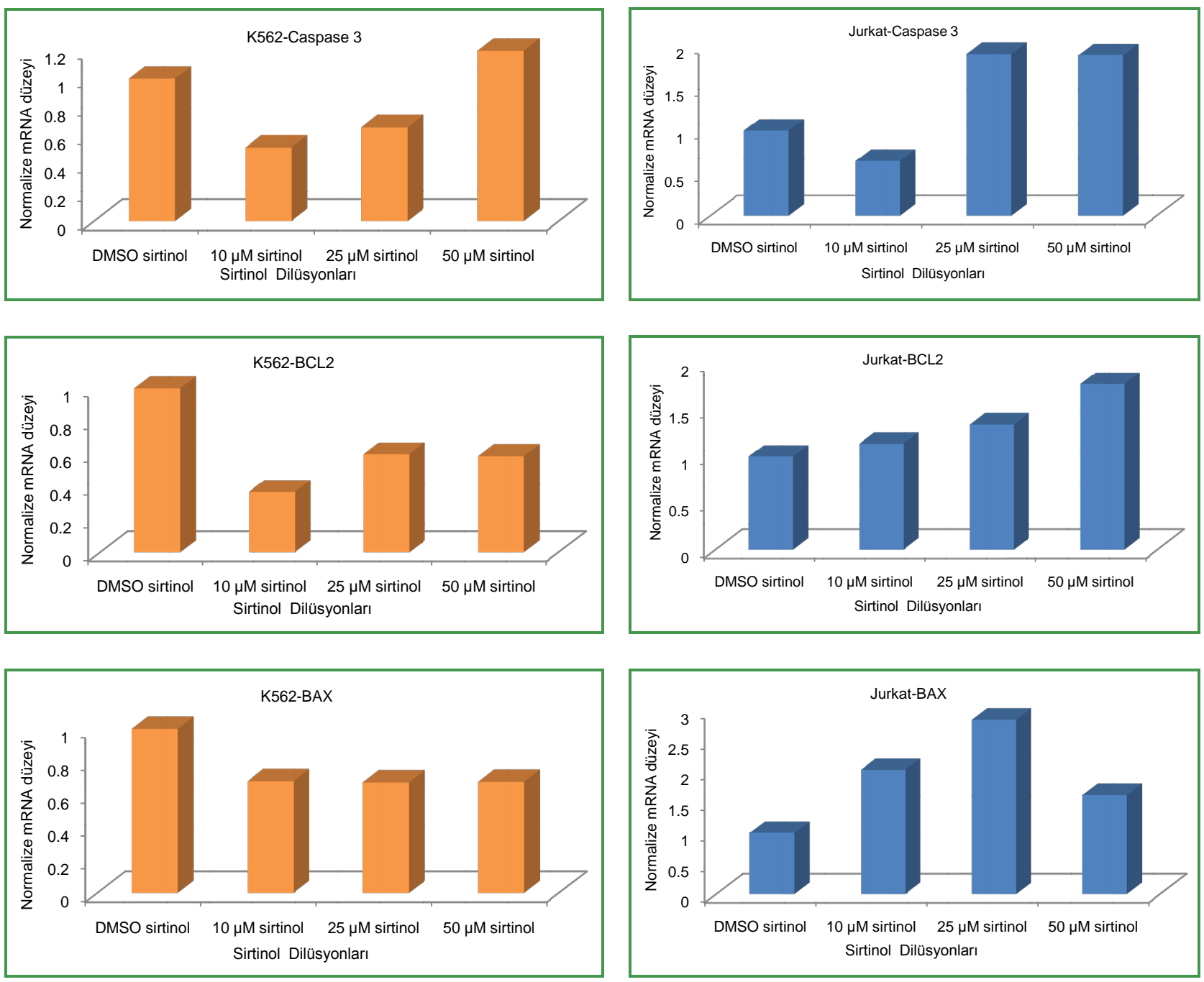

Figure 1. After Sirtinol application in K-562 and Jurkat cells, expression analysis of important genes involved in apoptosis was performed using real-time PCR. 
found to increase during other dilutions except $10 \mu \mathrm{M}$ level. It is observed that the $\mathrm{Bcl} 2$ expression increases with all dilutions and the Bax expression increases with the exception of $50 \mu \mathrm{M}$.

The increase in the ratio of $\mathrm{Bax} / \mathrm{Bcl} 2$ is considered to be the indicator of apoptosis. At K-562, an increase of Bax/Bcl2, especially at $10 \mu \mathrm{M}$, is observed. In Jurkat, it is observed that there is an increase of 10 and $25 \mu \mathrm{M}$ in this region (Figure 2).

Annexin- $\mathrm{V}$ staining was performed to determine the apoptosis-inducing effect of Sirtinol, and cells were analyzed by flow cytometry (Figure 3). As observed in the histograms, it is observed that in the Sirtinol dilutions which are increased in both K-562 and Jurkat, the number of living cells decreased and the number of dead cells increased, and this is peaking with a $50 \mu \mathrm{M}$ dilution.
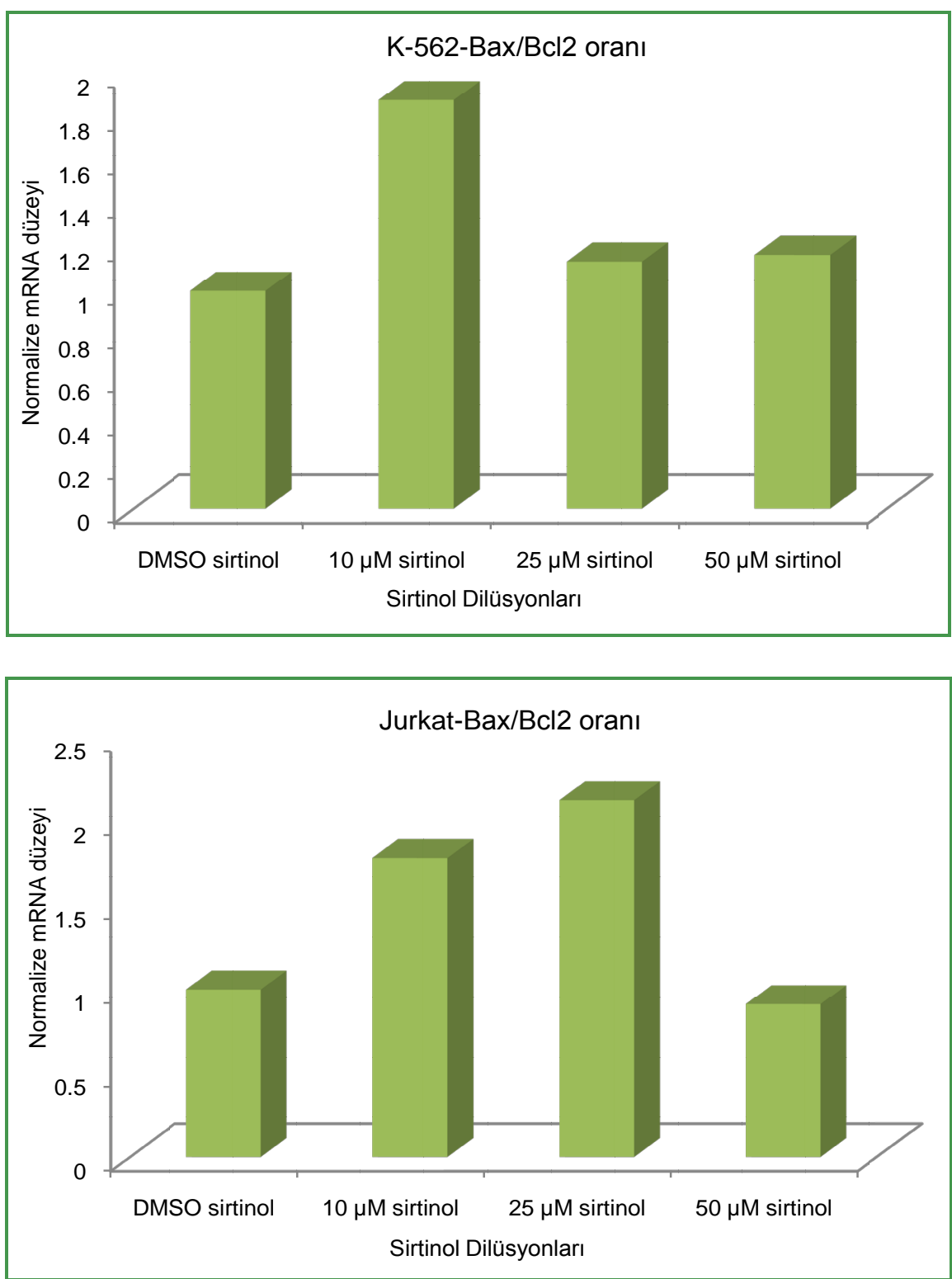

Figure 2. Analysis of Bax / Bcl2 ratio in cells after Sirtinol application in K-562 and Jurkat cells. Values were obtained by the proportion of normalized data obtained in real-time PCR. 

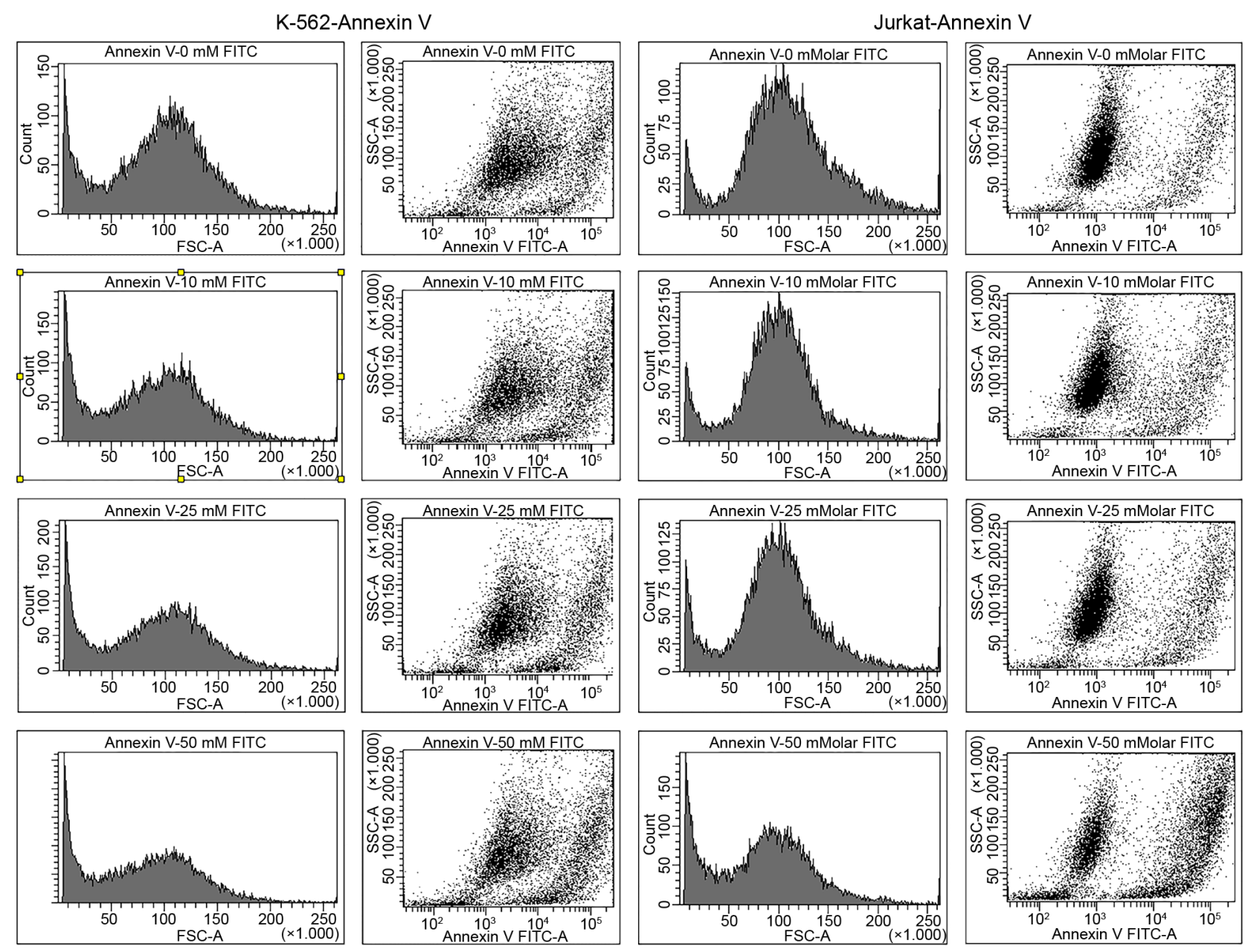

Figure 3. After Sirtinol application, apoptosis in K-562 and Jurkat cells was examined by flow cytometry using Annexin V method.

\section{Discussion}

Bax is an apoptotic pathway and Bcl-2 is an anti-apoptotic factor. The increase in $\mathrm{Bax} / \mathrm{Bcl} 2$ ratio is considered to be an important indicator for apoptosis. Caspase 3 is an important mediator of apoptosis. It has been shown in previous studies that Sirtinol apoptosed Jurkat and that Caspase3 was involved in this process [6]. However, the effect on Bax and Bcl2 was not studied. In K-562 cells, there is no information about the effect and mechanism of apoptosis in Sirtinol. The results obtained show that Sirtinol is apoptotic in Jurkat cells consistent with the literature, including Bax, Bcl2 and Caspase3. In the chronic myeloid cell line K562, Sirtinol induces apoptosis by inducing its effect at lower dilutions than Jurkat. Similar to Jurkat, we can say that the pathway including Bax, Bcl2 and Caspase 3 is activated and leads to apoptosis.

\section{Conclusion}

Sirtinol, an HDAC inhibitor in the direction of these results, has been observed to be a drug that can be effective in the treatment of CML as well as T-ALL. It will be aimed to investigate the mechanism of the next step in patient samples 
and investigate the target genes such as p53 and cMyc with different methods.

\section{References}

[1] Yuan, H., Su, L. and Chen, W.Y. (2013) The Emerging and Diverse Roles of Sirtuins in Cancer: A Clinical Perspective. Onco Targets and Therapy, 6, 1399-1416.

[2] Michishita, E., Park, J.Y., Burneskis, J.M., Barrett, J.C. and Horikawa, I. (2005) Evolutionarily Conserved and Nonconserved Cellular Localizations and Functions of Human SIRT Proteins. Molecular Biology of the Cell, 16, 4623-4635. https://doi.org/10.1091/mbc.E05-01-0033

[3] Liu, T., Liu, P.Y. and Marshall, G.M. (2009) The Critical Role of the Class III Histone Deacetylase SIRT1 in Cancer. Cancer Research, 69, 1702-1705. https://doi.org/10.1158/0008-5472.CAN-08-3365

[4] Fraga, M.F. and Esteller, M. (2007) Epigenetics and Aging: The Targets and the Marks. Trends in Genetics, 23, 413-418. https://doi.org/10.1016/j.tig.2007.05.008

[5] Lim, C.S. (2007) Human SIRT1: A Protein Biomarker for Tumorigenesis? Cell Bio logy International, 31, 636-637. https://doi.org/10.1016/j.cellbi.2006.11.003

[6] Kozako, T., Aikawa A., Shoji, T., Fujimoto, T., et al. (2012) High Expression of the Longevity Gene Product SIRT1 and Apoptosis Induction by Sirtinol in Adult T-Cell Leukemia Cells. T. International Journal of Cancer, 131, 2044-2055. https://doi.org/10.1002/ijc.27481

[7] Mumcuoğlu, M., Akın, D.F., Ezer, Ü. and Akar, N. (2015) Ankaferd Blood Stopper Induces Apoptosis and Regulates PAR1 and EPCR Expression in Human Leukemia Cells. Egyptian Journal of Medical Human Genetics, 83, 19-27.

https://doi.org/10.1016/j.ejmhg.2014.10.001 